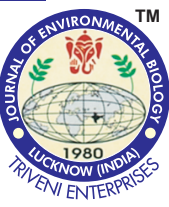

\title{
Characterization of glycolipid biosurfactant from Pseudomonas aeruginosa PA 1 and its efficacy against Phytophtora infestans
}

\begin{tabular}{lll}
\hline Paper received: 27.04.2018 Revised received: 23.09.2018 Re-revised received: 24.11.2018 Accepted: 10.01 .2019 & R
\end{tabular}

\section{Authors Info}

S. Tomar', M. Lal', M.A. Khan', B.P. Singh ${ }^{3}$ and S. Sharma ${ }^{3}$

${ }^{1}$ ICAR-Central Potato Research Institute-Regional Station, Meerut- 250 110, India

${ }^{2}$ ICAR- Indian Agricultural Research Institute, New Delhi-110 012, India

${ }^{3}$ ICAR- Central Potato Research Institute, Shimla -170 001, India

*Corresponding Author Email : mehilalonline@gmail.com

\section{Edited by \\ Dr. R.B. Raizada}

Reviewed by

Dr. Harsh Vardhan Singh

Dr. Neelam Garg

\section{Abstract}

Aim : The aim of the present study was to identify biosurfactant produced by Pseudomonas aeruginosa and to determine the effectiveness of biosurfactants (rhamnolipid) against $P$. infestans causing late blight of potato.

Methodology : Pseudomonas aeruginosa strains were isolated from soil samples and the potential strain PA 1, selected because of its antagonistic ability, was used to optimize anti-metabolite production and its characterization using HPLC-Mass spectrometry. Rhamnolipid based formulation was developed and its efficacy was tested against late blight disease.

Results : The results revealed that four rhamnolipids congeners were identified, among them three were mono-rhamno-di-lipidic congeners and one was di-rhamno-di-lipdic congeners, abundantly present in the crude biosurfactant obtained from $P$. aeruginosa PA1. The mass spectra of mono-rhamno-di-lipidic Rha- $\mathrm{C}_{12}-\mathrm{C}_{14}$ peak value ( $\left.\mathrm{m} / \mathrm{z} 584\right)$, Rha- $\mathrm{C}_{12.1}-\mathrm{C}_{10 . \mathrm{CH} 3}$ peak value $(\mathrm{m} / \mathrm{z}$

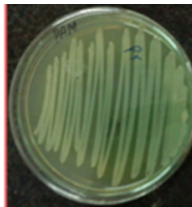

Isolated $P$. aeruginosa culture

Rhamnolipid-based formulation was effective at $0.2 \%$ concentration for management of late blight of potato

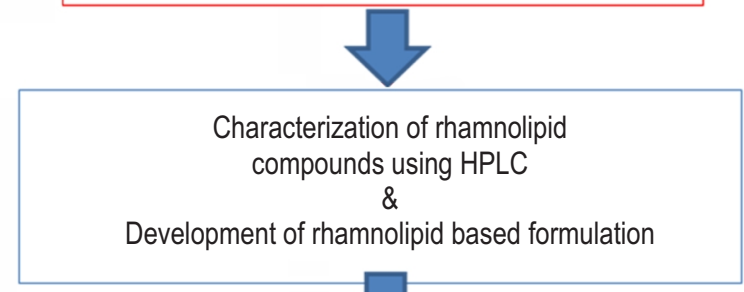

Evaluation of effect of rhamnolipid based formulation against late blight lesions in detached leaf test in detached leaf test

545), Rha- $\mathrm{C}_{12}-\mathrm{C}_{-12 \text {-снз }}$ peak value (m/z 575) and di-rhamno-di-lipidic. Rha-Rha- $\mathrm{C}_{10}-\mathrm{C}_{10}$ peak value ( $\left.\mathrm{m} / \mathrm{z} 651\right)$ were also detected. Rhamnolipid-based formulation was developed and evaluated at different concentration ranging from 0.012 and $0.3 \%$ in detached leaf test. Significant reduction in lesion area was recorded at $0.2 \%$ concentration (lesion area $0.06 \mathrm{~cm}^{2}$ as against $9.8 \mathrm{~cm}^{2}$ on $5^{\text {th }}$ day of inoculation).

Interpretation : Microbial produced rhamnolipid based formulation at $0.2 \%$ concentration was found effective against late blight of potato in detached leaf test. Further, it could be used in field study as green chemical which would help in replacing application of chemicals in agriculture.

Key words: Biosurfactant, Late blight disease, Phytophthora infestans, Potato, Rhamnolipid

How to cite : Tomar, S., M. Lal, M.A. Khan, B.P. Singh and S. Sharma: Characterization of glycolipid biosurfactant from Pseudomonas aeruginosa PA 1 and its efficacy against Phytophtora infestans. J. Environ. Biol., 40, 725-730 (2019). 


\section{Introduction}

Potato is one of the most important food crop of the world. It ranks third after rice and wheat in the world (Rajiv and Kawar 2016). Potato crop is affected by various diseases like late blight, early blight and leaf spot complex, black scurf, common scab, bacterial wilt and viral diseases. Among these diseases, late blight caused by Phytophthora infestans (Mont.) de Bary, is considered to be the most serious and widely distributed disease across the world. The disease severity goes up to $100 \%$ and maximum yield loss occurs, if the crop is attacked at early stage. An average $10-15 \%$ yield loss of potato has been reported due to late blight in India (Lal et al., 2016). This disease is being managed by adopting chemical, biological methods and using resistant varieties. Although, chemical management is a good option, however, due to hazardous nature of chemicals, pathogen develop resistance against chemicals and also has residua concern. Therefore, it is not suitable for continuous use for controlling the disease. Host resistance is broken down due to matching of virulence genes in $P$. infestans over a period.

Biological control can be utilized to manage the disease for optimum potato yield with safe production. Biosurfactants are produced by bacteria, yeasts and fungi that serve as green surfactants. Moreover, biosurfactants are potentially active stable and exhibit low toxicity. It has wide variety of structures and can be produced from renewable feed-stocks by using wide variety of microorganisms. Most importantly, they are biodegradable, environmental friendly and are used as green chemicals in the agricultural industry (Dhara et al., 2013). Biosurfactants are classified, according to their molecular structure, mainly as glycolipids i.e., rhamnolipid (RLS) and sophorolipids, lipopeptides (surfactin), polymeric biosurfactants (emulsan and alasan), fatty acids (3-(3-hydroxyalkanoyloxy) alkanoic acids (HAAs) and phospholipids (phosphatidylethanolamine). Rhamnolipids are further classified as mono-rhamnolipids and di-rhamnolipids, depending on one or two rhamnose sugars linked to a dimer of $\beta$-hydroxyacids (primarily $\beta$-hydroxydecanoate) which can rise up to three hydroxyl fatty acids containing 8-14 carbons (Heyd et al., 2008).

The potential of various rhamnolipid producing fluorescent $P$ seudomonas spp. have been evaluated in-vitro and in-vivo systems against Phytophthoracapsici on pepper crop (Özyilmaz and Kemal, 2013). Bengtsson et al. (2015) and Demeulenaere and Hofte (2000) reported that the surfactants exhibit lytic activity against zoospores of the pathogens. Pseudomonas strains are the best producers of glycolipid containing rhamnose and 3-hydroxy fatty acids. The most promising bacterial strains, $P$. aeruginosa, have been recommended as the best microorganism to produce two classes of rhamnolipids: monorhamnolipids and dirhamnolipids with excellent surface activity (Irfan-Maqsood and Seddiq-Shams, 2014). Rhamnolipids have both lyophilic and lyophobic group which facilitates the orientation of surfactants to reduce the liquid surface free energy and increase surface viscosity (Mao et al., 2015; Gujar and Hamde, 2012). The research aspects on microbial metabolite production and their applicability in controlling plant diseases is gaining momentum in agriculture. The scope of developing microbial metabolites for commercial pesticides as an alternative to chemical fungicides is important due to increased concern on environmental pollution, pathogen resistance and high plant protection costs. In view of the above, objective of the present study was to identify the biosurfactant produced by Pseudomonas aeruginosa and to determine the effectiveness of biosurfactants against $P$. infestans.

\section{Materials and Methods}

Isolation of $P$. infestans: $P$. infestans was isolated using sodium hypochlorite $(0.25 \%)$ from potato late blight infected tubers, stems and leaves on Rye B medium (rye $60 \mathrm{~g}$, sucrose 15 $\mathrm{g}$, agar $15 \mathrm{~g}$, water $1000 \mathrm{ml}$ antibiotics, rifampicin @ $0.02 \mathrm{gl}^{-1}$ and ampicillin @ $0.10 \mathrm{~g} \mathrm{l}^{-1}$ ). The isolated culture was purified using hyphal tip method and identified using compound microscopy. The isolated culture was maintained on Rye B medium and kept at $10^{\circ} \mathrm{C}$ for further use.

Isolation and in-vitro screening of Pseudomonas aeruginosa against late blight: Rhizospheric soil samples were collected from different regions of India (Northern: Western UP, Delhi, Haridwar and Shimla, Southern: Hyderabad and Chick Mangalore and Eastern: Eastern UP Gorakhpur) including oil spill sites and strains of Pseudomonas aeruginosa were isolated on King's B medium. These identified strains were used for further studies and screened in-vitro for their antagonistic ability against late blight pathogen, $P$. infestans by dual culture method and their anti-metabolite were evaluated in food poisoning test.

Standardization of glycolipid biosurfactant production method: $P$. aeruginosa PA 1 strain was selected because of its superior antagonistic ability and was used to optimize antimetabolite production. Nutrient broth plus glycerol as carbon source was used for the growth of PA 1 in conical flask at $180 \mathrm{rpm}$ and $28^{\circ} \mathrm{C}$ on rotatory shaker for 12 days. Active cells were centrifuged, extracted and evaporated. The final product, a dark brown thick paste, was run on TLC plates which showed yellow spot for initial confirmation.

Characterization of glycolipid biosurfactant through HPLCMass Spectroscopy method: The HPLC-MS of extracted crude biosurfactant was done on MS system consisting of Waters HPLC model (ACQ-TQD=QBB 1152) Waters Acquity SDS method, using a gradient elution high-performance liquid chromatography (HPLC) with a dual absorbance UV detector, fitted with C18 column having a dimension of $100 \times 2.1 .1 .7 \mu \mathrm{m}$, and Waters Acquity PDA method, UPLC, LG-500 nm, was used. The mobile phase was kept at a flow ramp rate of $0.45 \mathrm{ml} \mathrm{min}^{-1}$ and the sample injection volume was $10 \mu \mathrm{l}$. The mobile phase solutions were acetonitrile/water (5:95, v/v) (A), Acetonitrile (B), Methanol (C) and $5 \mathrm{mM}$ ammonium acetate of $\mathrm{pH} 6.5$ buffer (D). Run time was $20 \mathrm{~min}$, Range - $200-450 \mathrm{~nm}$, resolution $1.2 \mathrm{~nm}$. MS scan was started at 150 mass and ended at 1150 mass under $\mathrm{ESI}^{+}$source and MS-MS collision energy was 20.0. All eluted 
peaks from HPLC were recorded at different retention times. The fractions were then characterized by mass spectrometry. This analysis was carried out at Sophisticated Analytical Instrumentation Facility (SAIF), CSIR- Central Drug Research Institute (CDRI), Lucknow (U.P).

Development of rhamnolipid formulation and its evaluation against late blight: Rhamnolipid-based formulation (25\%) was developed by mixing $25 \mathrm{~g}$ of crude biosurfactant containing a mixture of mono and di-rhamnolipid in $100 \mathrm{ml}$ mixture of cyclohexanone $(99.5 \mathrm{ml})$ and tween-80 $(0.5 \mathrm{ml})$. The formulation was evaluated against $P$. infestans using detached leaf assay. Potato plants of Cv. Kufri Bahar, were grown in glass house. Leaves were plucked (fourth from the top) and treated with rhamnolipid-based formulation at different concentrations $(0.012$, $0.25,0.05,0.075,0.1,0.15,0.2,0.25$ and $0.3 \%$ ) prior to Zoospores inoculation on leaves. Water and cyclohexanone $(3.0 \%)$ treated leaves served as control. Cyclohexane was part of formulation; therefore, its effect was compared as control. The treated leaves were dried and inoculated with zoospores suspension $\left(6 \times 10^{4}\right.$ per $\left.\mathrm{ml}\right)$ at the abaxial side of the leaflet. To avoid evaporation of droplet, the leaflets were placed on a net in a plastic tray containing moist filter paper. Tray containing treated and untreated leaflets (control) were incubated for five days in growth chamber at $18 \pm 1^{\circ} \mathrm{C}$ and $>95 \% \mathrm{RH}$. The lesion area was measured by the formula of Singh and Bhattacharyya (1995). The experiment was conducted using Completely Randomized Design (CRD) and data were subjected to statistical analysis with the help of IRRISTAT software (version 4.4.20030719).

\section{Results and Discussion}

$P$. infestans were isolated from late blight infected potato leaves, stems and tubers. The isolate was purified and identified using sporangial characteristics, i.e., pear shaped papilate sporangia. The identified fungal isolates was maintained on Rye Agar $\mathrm{B}$ medium at $10 \pm 1^{\circ} \mathrm{C}$ and used in all the studies. Bacterial cultures were isolated from oil spill sites, rhizospheric soils and identified as $P$. aeruginosa based on molecular data (Tomar et al., 2013). P. aeruginosa isolate, which showed best inhibition percentage in dual culture and food poisoning test (results not shown), was selected for biosurfactant production and characterization. Studies on standardization of glycolipid production from $P$. aeruginosa showed that nutrient broth supplemented with $2 \%$ glycerol was suitable medium for production of glycolipid biosurfactants. The yellow spot on TLC plate showed the presence of glycolipid group of biosurfactants in the dark brown liquid obtained after extraction. HPLC chromatogram showed several compounds on the chromatogram (Fig.1). Four of these compounds were identified as rhamnolipids congeners based on

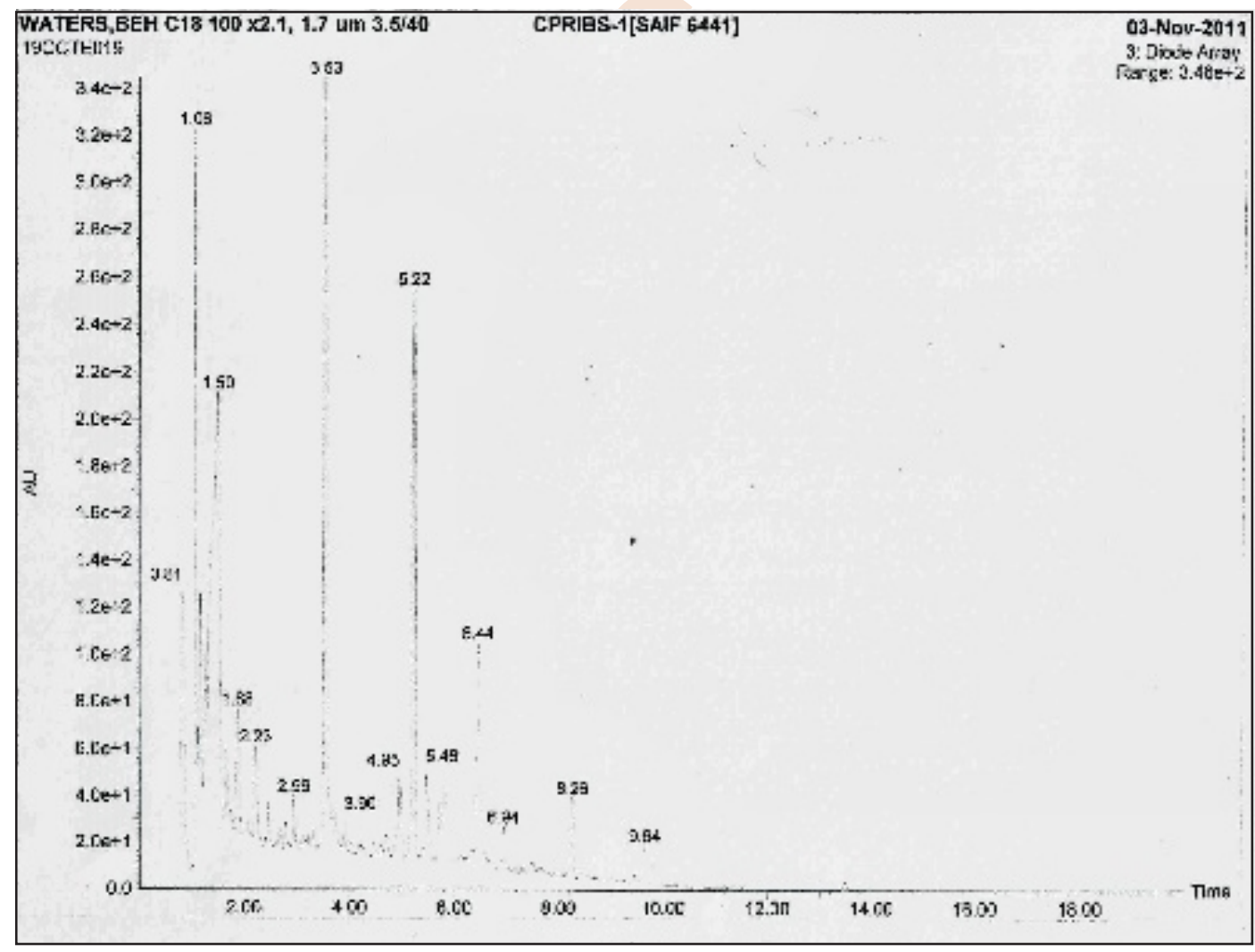

Fig.1: HPLC chromatogram of crude biosurfactant obtained from P. aeruginosa. 
Table 1: Molecular formula, molecular weight, mass to charge ratio and structure of different rhamnolipids identified from P. aeruginosa using LC-MS

\begin{tabular}{|c|c|c|c|c|c|c|c|c|}
\hline Symbol & $\begin{array}{l}\text { Molecular } \\
\text { formula }\end{array}$ & $\begin{array}{l}\text { Molecular } \\
\text { weight }\end{array}$ & $\begin{array}{l}\mathrm{m} / \mathrm{z} \\
\text { obtained }\end{array}$ & R1 & $\mathrm{n} 1$ & $\mathrm{n} 2$ & $\mathrm{R} 2$ & Structure \\
\hline Rha-C12-C14 & $\mathrm{C}_{32} \mathrm{H}_{60} \mathrm{O}_{9}$ & 588 & $584 . .6$ & $\mathrm{H}$ & 5 & 7 & $\mathrm{H}$ & \\
\hline Rha-C12.1-C10-CH3 & $\mathrm{C}_{29} \mathrm{H}_{63} \mathrm{O}_{9}$ & 545 & 545.5 & $\mathrm{H}$ & 3 & - & $\mathrm{CH}_{3}$ & \\
\hline Rha-C12-C12-CH3 & $\mathrm{C}_{31} \mathrm{H}_{59} \mathrm{O}_{9}$ & 574 & 574.6 & $\mathrm{H}$ & - & - & $\mathrm{CH}_{3}$ & \\
\hline Rha-Rha-C10-C10 & $\mathrm{C}_{32} \mathrm{H}_{58} \mathrm{O}_{13}$ & 650 & 651.8 & $\mathrm{H}$ & - & $\mathrm{H}$ & $\mathrm{H}$ & \\
\hline Unknown & * & * & * & * & * & * & * & b: a-decenoyl grc \\
\hline Unknown & * & * & * & * & * & & & \\
\hline Unknown & * & * & * & * & * & * & & \\
\hline Unknown & * & * & * & * & * & * & * & \\
\hline
\end{tabular}

their molecular formula and molecular weights (Table 1). Among all four rhamnolipids congeners, three were mono-rhamno-di-lipidic congeners and one was di-rhamno-di-lipdic congener, present in abundance in the crude biosurfactant obtained from $P$. aeruginosa. The mass spectra of mono-rhamno-di-lipidic Rha- $C_{12}-C_{14}$ peak value (m/z 584), Rha- $C_{12.1}-C_{10 . \text { Сн3 }}$ peak value (m/z 545), Rha- $C_{12}-C_{-12}$ снз peak value ( $\mathrm{m} / \mathrm{z} 575)$ and di-rhamno-di-lipidic Rha-Rha- $\mathrm{C}_{10}-\mathrm{C}_{10}$ peak value ( $\mathrm{m} / \mathrm{z} 651$ ) is shown in Fig. 2 (a-d). Other compounds with their peak values $(682.8,681.9,708.8$ and 935.2) could not be identified since no matching real value were available in literature. Hence, LC-MS analysis confirmed that the rhamnolipid produced by $P$. aeruginosa is a mixture of different rhamnolipidic congeners (mono-rhamno-di-lipidic and di-rhamno-di-lipidic) and the monorhamno-di-lipidic congener had the highest relative abundance of $\mathrm{M}^{-}$and $[\mathrm{M}-\mathrm{H}]^{-}$. The results of the present study is supported by initial discovery of rhamnolipids (RLs) by Bergstrom et al. (1946). Milena et al. (2015) reported that glycolipids produced by $P$ seudomonas pyocyanea ( $P$. aeruginosa) are known as pyolipic acid whose structural units were identified as L-rhamnose and $\beta$ hydroxydecanoic acids. High-performance-liquid chromatography method used to quantify rhamnolipids in a bacterial biosurfactant mixture of $P$. aeruginosa strain and two rhamnolipids, i.e., Rha C10-C10 and Rha-Rha C10-C10 were identified (Raza et al., 2009). Two additional RL congeners were identified in cultures of Pseudomonas sp. DSM 2874 grown as resting cells on n-alkanes or glycerol. These mono-lipidic RLs were also detected in trace amounts in continuous $P$. aeruginosa cultures (Sinumvayo and Ishimwe, 2015).

Rhamnolipid based liquid formulation was evaluated for its efficacy against $P$. infestans, using detached leaf method. The results revealed a statistically significant $(P<0.05=0.34, S E=$
0.11 for $4^{\text {th }}$ day and $\mathrm{P}<0.05=0.35, \mathrm{SE}=0.12$ for $5^{\text {th }}$ day) reduction in lesion area of late blight disease when leaves were treated with $0.2 \%$ of formulation. Treated leaves showed lesion area of $0.06 \mathrm{~cm}^{2}$ as compared to control $\left(9.8 \mathrm{~cm}^{2}\right)$ on $5^{\text {th }}$ day of inoculation. However, at lower concentration $(0.15 \%$ and below), a lesion area of $1.5 \mathrm{~cm}^{2}$ and more was observed (Fig. 3). At highest concentration $(0.3 \%)$, slight phytotoxicity was observed on detached leaf test. Three percent cyclohexane treated (control) leaves did not control the disease because the lesions were similar to control (with water only). As cyclohexane is a part of liquid formulation, its role was also evaluated as control treatment for reducing the lesion area of the disease.

This study reveals that it is possible to obtain significant reduction in development of late blight disease in potatoes after treatment with rhamnolipid. Rhmanolipids are biosurfactants, generally known to be less toxic compared to synthetic surfactants and are rapidly degraded in the environment (Lima et al., 2011; Mulligan, 2005). Bioformulation of rhmanolipids used in this study showed better inhibition at $0.2 \%$ concentration, and no sign of phytotoxicity was observed at this concentration. However, at $0.3 \%$ concentration a slight toxicity was observed in potato cultivar K. Bahar leaves while performing detached leaf test. Similar results were observed when leaves of potato plants treated with $0.5 \mathrm{mg} \mathrm{ml}^{-1}$ biosurfactant showed no signs of phytotoxicity on the leaves (Hultberg et al., 2010). However, phytotoxicity due to leaf application of Triton-X surfactants has also been reported (Bukovac, 2005), which may be due to higher doses of surfactant and other climatic factors. The ability of a biosurfactant to reduce disease in tomatoes and potatoes late blight caused by $P$. infestans has been reported by Tran et al. (2007); Martinez and Osorio (2007). One of the possible mode of action of biosurfactants 


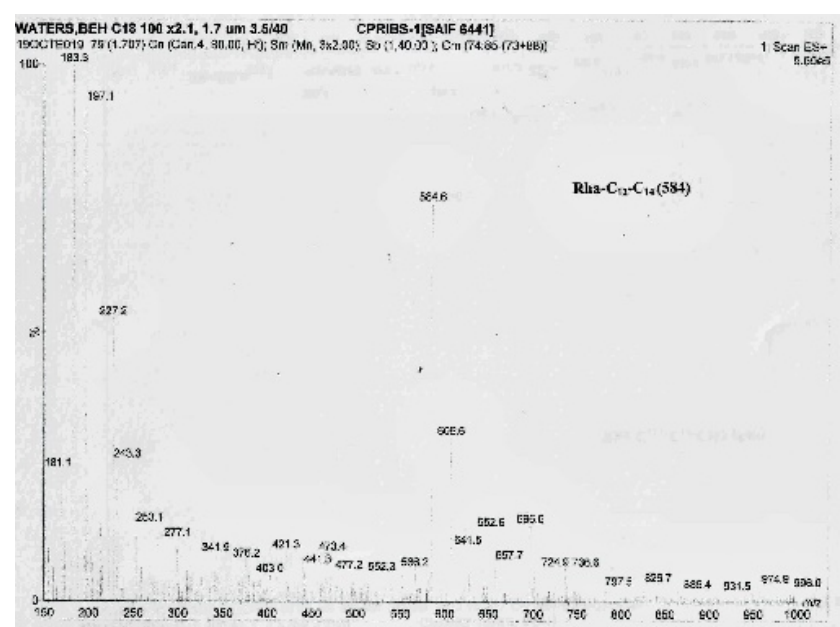

(a)

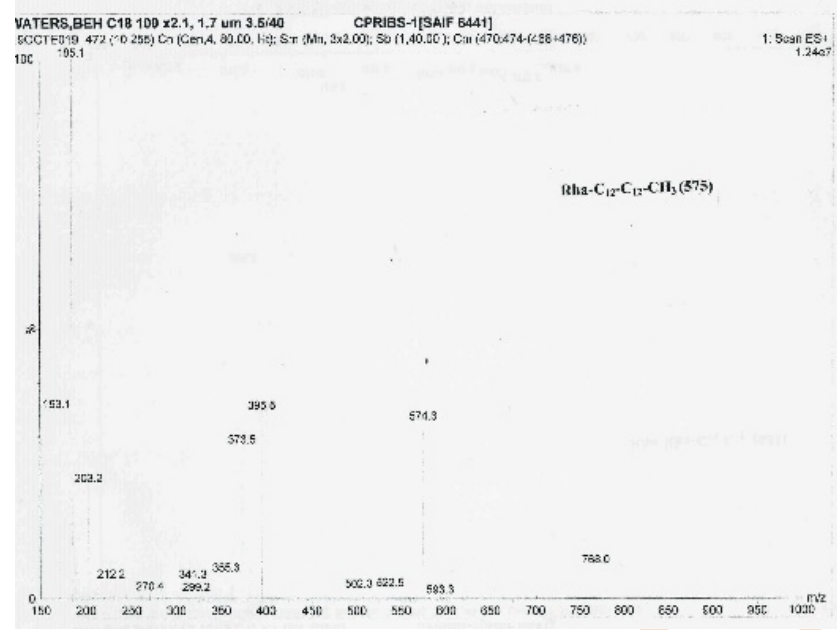

(c)

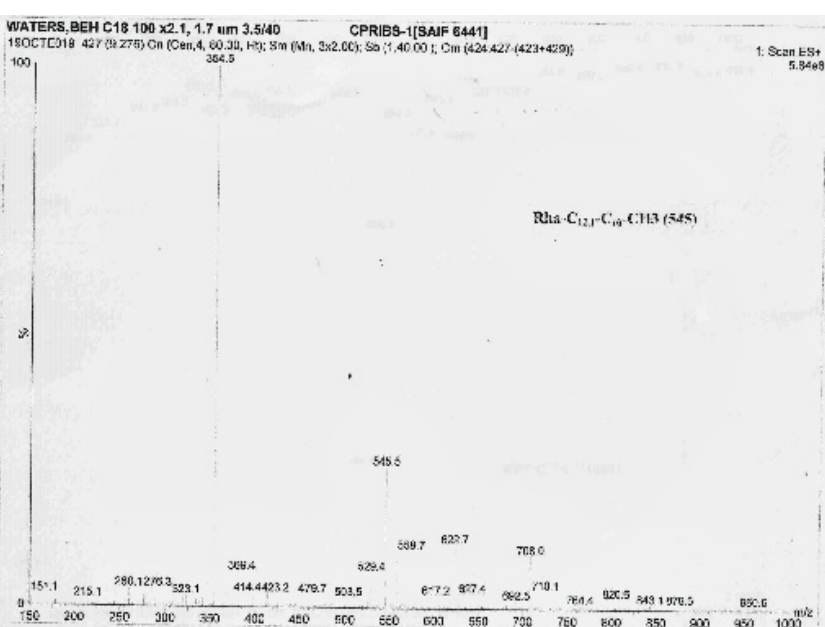

(b)

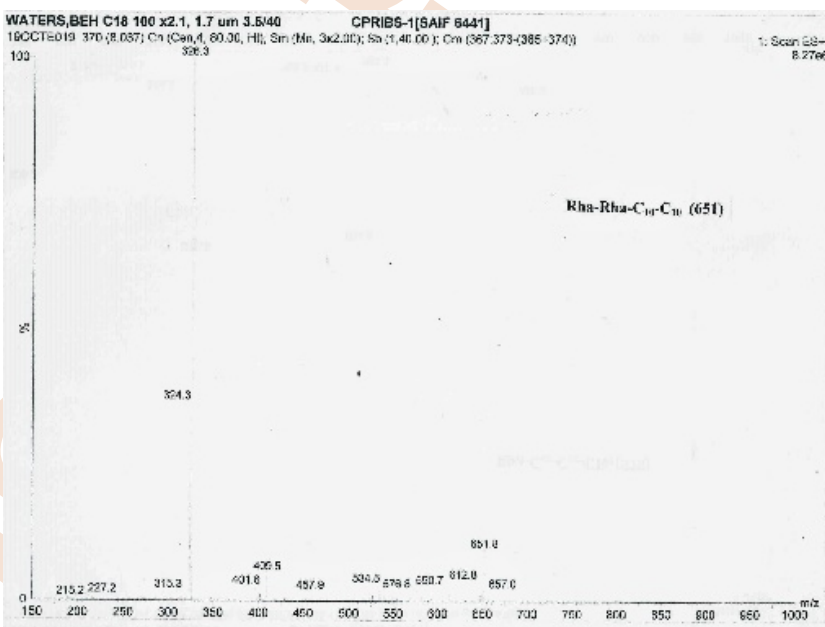

(d)

Fig. 2: LC-MS chromatogram of crude biosurfactant of $P$. aeruginosa: (a) Rha- $\mathrm{C}_{12}-\mathrm{C}_{14}(584)$; (b) Rha- $\mathrm{C}_{12.1}-\mathrm{C}_{10}-\mathrm{CH}_{3}(545)$; (c) Rha- $\mathrm{C}_{12}-\mathrm{C}_{12}-\mathrm{CH}_{3}(575)$ and (d) Rha-Rha- $\mathrm{C}_{10}-\mathrm{C}_{10}(651)$.

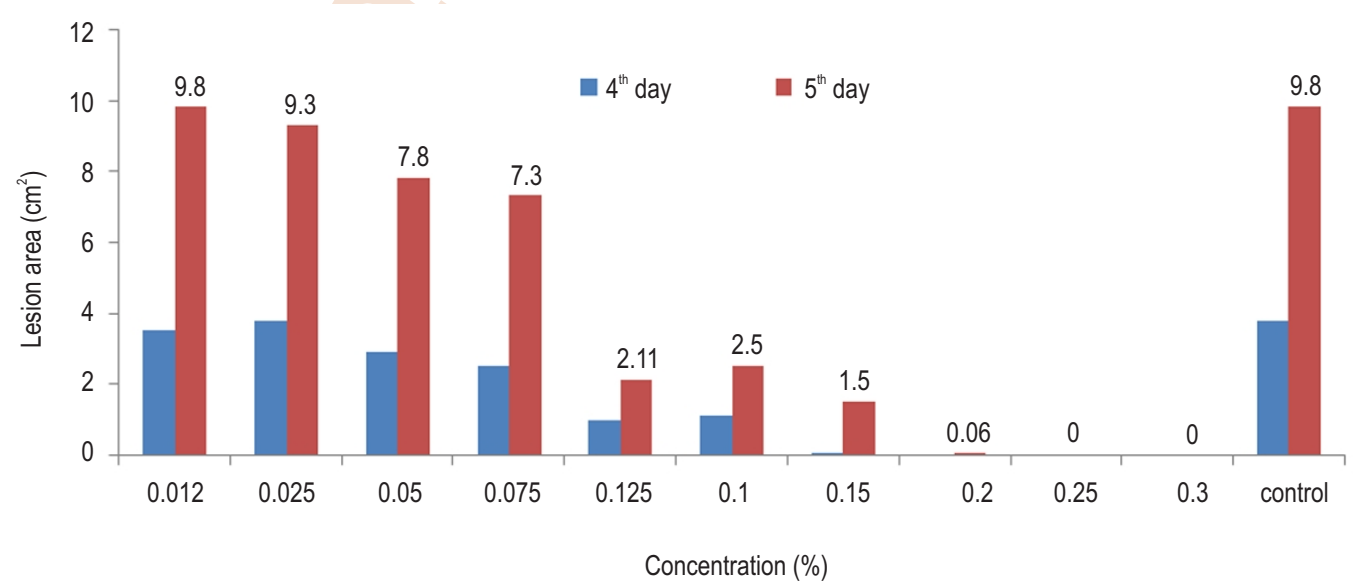

Fig. 3: Effect of rhamnolipid-based formulation on late blight lesion developed on potato leaves. 
on zoosporic plant pathogens is destabilisation of membranes, which causes lysis of zoospores.

In the present study, potato leaves were pre-treated with rhamnolipid formulation before inoculating zoospore suspension. A significant reduction in lesion area and consistent effect of more than five day period was observed in the experiments on direct exposure of zoospores to biosurfactant (rhamnolipid formulation) indicates that zoospores are most probably lysed by biosurfactant treatment. Lysis of zoospore exposed to biosurfactant has been visually demonstrated with massetolide $A$ and other rhamnolipds (Stanghellini and Miller, 1997; De Souza et al., 2003; Soltani et al., 2016). Biosurfactants produced by fluorescent pseudomonads have zoospore lysis activity and biosurfactant-producing strain Pseudomonas koreensis 2.74 has potential to induce resistance in potato plant against late blight disease (Bengtsson et al., 2015). High sensitivity of $P$. infestans zoospores to biosurfactants suggest that they can be used to dampen the spread of potato late blight once infection has been detected in the field. At present study four rhamnolipids congeners were identified, among them three were mono-rhamno-di-lipidic congeners and one was dirhamno-di-lipdic congeners present in abundance in the crude biosurfactant obtained from $P$. aeruginosa PA1. Rhamnolipidbased liquid formulation $0.2 \%$ developed from $P$. aeruginosa PA1 was effective in the management of late blight of potato in detached leaf test. Further, rhamnolipid based formulation can be used by farmers, after proper field evaluation, for the management of late blight of potato.

\section{Acknowledgments}

The authors are thankful to ICAR New Delhi for providing fund through Outreach project on Phytophthora, Fusarium and Ralstonia diseases of horticultural and field crops (PhytoFura). The authors are also grateful to the Sophisticated Analytical Instrumentation Facility (SAIF), CSIR- Central Drug Research Institute (CDRI) Lucknow, for HPLC analysis.

\section{References}

Bengtsson, T., E. Liljeroth, E. Andreasson, A. Holefors and M. Hultberg: Biosurfactants have the potential to induce defence against Phytophthora infestans in potato. Potato Res., 58, 83-90 (2015).

Bergström, S., H. Theorell and H. Davide: On a metabolic product of $P$. pyocyanea. Pyolipic acid, active against M. tuberculosis. Arkiv. Chem. Mineral. Geol., 23 A, 1-12 (1946).

Bukovac, M.J.: Maximizing performance of plant growth regulators by improving spray application. Hort. Technology, 15, 222-23 (2005).

De Souza, J.T., M. de Boer, P. de Waard, T. A. Van Beek and J. M. Raaijmakers: Biochemical, genetic and zoosporicidal properties of cyclic lipopeptide surfactants produced by Pseudomonas fluorescence. Appl. Environ. Microbiol., 69, 7161-7172 (2003).

Demeulenaere, $\mathrm{N}$. and M. Hofte: Influence of several surfactants on various asexual stages in life cycle of two phytophthora species. In: Pests and Diseases Farnham, UK: BCPC Pulications, pp. 257-260 (2000).

Dhara, P., S. Sachdev and S. Cameotra: Biosurfactants in agriculture. Appl. Microbiol. Biotechnol., 97,1005-1016 (2013).
Gujar, R.S. and V.S Hamde: Separation and characterization of biosurfactant from $P$. aeruginosa sp1 isolated from oil mill area MIDC, Parbhani (M.S.). Int. Multidiscip. Res. J., 2, 13-15 (2012).

Heyd, M., A. Kohnert, T.H Tan, M. Nusser, F. Kirschhöfer, G. B. W. Franzreb and M. S. Berensmeier: Development and trends of biosurfactant analysis and purification using rhamnolipids as an example. Anal. Bioanal. Chem., 391, 1579-90 (2008).

Hultberg, M., T. Bengtsso and E. Liljeroth: Late blight on potato is suppressed by the biosurfactant producing strains Pseudomonas koreensis 2.74 and its biosurfactant. BioControl, 55, 543-550 (2010).

Irfan-Maqsood, M. and M. Seddiq-Shams: Rhamnolipids well characterized glycolipids with potential broad applicability as biosurfactants. Ind. Biotechnol., 10, 285-291(2014).

Lal, M., R.KArora, U. Maheshwari, S. Rawal and S. Yadav: Impact of late blight occurrence on potato productivity during 2013-14. Int. J. Agricult. Stat. Sci., 12, 187-192 (2016).

Lima, T.M., L.C. Procopio, F.D. Brandao, A.M. Caralho, M.R. Totola and A.C. Borges: Biodegradabiity of bacterial surfactants. Biodegradation, 22, 585-592 (2011).

Mao, X., R. Jiang, W. Xiao and J. Yu: Use of surfactants for the remediation of contaminated soils: A review. J. Hazard. Mater., 285, 419-435 (2015).

Martinez, E.P. and J.A. Osorio: Preliminary studies for the production of an active biosurfactant against Phytophthora infestans (Mont.) de Bary. Revista Corpoica Ciencia Technologia Agropecuarias, 8, 5-16 (2007).

Milena, G. R., M. V. Miroslav and M. K. J. Ivanka: Rhamnolipid biosurfactant from Pseudomonas aeruginosa-From discovery to application in contemporary technology. Serb. Chem. Soc., 80, 279-304 (2015).

Mulligan, C.N.: Environmental application for biosurfacatnts. Environ. Pollution., 133, 183-198 (2005).

Özyilmaz, Ü. and B. Kemal: Enhanced biological control of Phytophthora blight of pepper by biosurfactant-producing Pseudomonas. Plant Pathol. J., 29, 418-426 (2013).

Rajiv and P.G.Kawar: Enriched potato for mitigating hidden hunger. In: Biofortification For Food Crops (Eds.: U. Singh, C.S. Praharaj, S.S. Singh and N.P. Singh). Springer India, p.433-457 (2016).

Raza, Z.A., Z.M. Khalid and I.M. Banat: Characterization of rhamnolipids produced by a Pseudomonas aeruginosa mutant strain grown on waste oils. J. Environ. Sci. Hlth. Tox. Hazard. Subst. Environ. Eng., 44, 1367-73(2009).

Singh, B.P. and S.K. Bhattacharyya: Field resistance to late blight in four Indian potato cultivars. Potato Res., 38,171-178 (1995).

Sinumvayo, J.P. and N. Ishimwe: Agriculture and food applications of rhamnolipids and its production by Pseudomonas aeruginosa. J. Chem .Eng. Process. Technol., 6, 1000223 (2015).

Soltani, D. S., J. Kohl and L. Ju: Rhamnolipid adsorption in soil: Factors, unique features, and considerations for use as green antizoosporic agents. J. Agr. Food. Chem., 64, 3330-3337 (2016).

Stanghellini, M.E. and R.M. Miller: Biosurfactant: Their identity and potential efficacy in the biological control of zoosporic plant pathogens. Plant Dis., 81, 14-12(1997).

Tomar, S., B.P. Singh, M.A. Khan, S. Kumar, S. Sharma and M. Lal: Identification of Pseudomonas aeruginosa strain producing biosurfactant with antifungal activity against Phytophthora infestans. Pot. J., 40, 155-163 (2013).

Tran, H., A. Ficke, T. Asiimwe, M. Hofte and J.M. Raaijmakers: Role of the cyclic lipopeptide massetolide a in biological control of Phytophthora infestans and in colonization of tomato plants by Pseudomonas fluorescence. New Phytologist, 175, 731-742 (2007). 